Editorial (Special Column for the Youth Innovation Promotion Association, Chinese Academy of Sciences)

\title{
Preface to Special Column for the Youth Innovation Promotion Association, Chinese Academy of Sciences
}

The Youth Innovation Promotion Association (abbreviated as YIPA) of the Chinese Academy of Sciences (abbreviated as CAS) was established in 2011. It is an innovative measure for the comprehensive training of young science and technology talents under the age of 35 by the CAS. The YIPA aims to organize and support the academic exchange and cooperation of young researchers of the CAS and to train a new generation of academic and technical leaders. By the end of 2018, the number of members of the YIPA had reached 3640, which is the core backbone of the young researchers of the CAS. The YIPA has a total of six discipline branches: mathematics science, chemistry and materials, life sciences, earth sciences, information and management, engineering and equipment.

The Chemistry and Materials Branch (CMB for short) is an important subject organization in YIPA. At present, the CMB has nearly 900 members from 22 institutes and universities of the CAS. During the past years, CMB has carried out a series of pioneering work. These efforts have played a very good role in uniting young scientists and technicians in various units, improving rules and regulations, strengthening academic exchanges, creating distinctive brand activities, promoting interdisciplinary and integration of disciplines, cultivating outstanding talents, and promoting the transfer of scientific and technological achievements. In order to fully demonstrate the latest research progress and achievements of the members of the CMB in the field of catalysis, the Chinese Journal of Catalysis and the CMB jointly published the special Column. Researcher Qingde Zhang, the president of the CMB, and Hongzhang Zhang, the director of the Council of the CAS, were invited to serve as the guest editors of this special column.

This special column has published 10 papers, including 3 reviews, 1 newsletter, and 6 research papers covering the latest research progress in energy, environment, new materials, heterogeneous catalysis, and surface chemistry. The publication of this special column will further enhance the academic influence of the members of the Association for the Advancement of Science in the field of catalysis, and at the same time strengthen the vitality of the Chinese Journal of Catalysis.

Special thanks to Academician Can Li and Academician Tao Zhang, Editors-in-Chief of the Chinese Journal of Catalysis, for their guidance and support for this special column!

We are very grateful to all members of the Second Committee of the CMB for their support of this special column. We sincerely thank all the members and related authors who have provided high-level papers. Thanks to the reviewers for their valuable comments and suggestions for improving the qulity of the articles. We thank the editorial staff for their hard work in the successful publication of the special column.

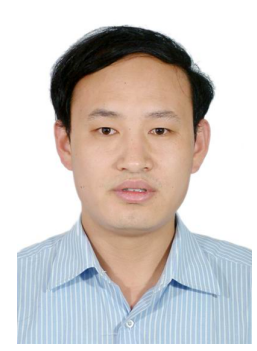

Prof. Qingde Zhang (Guest Editor)

State Key Laboratory of Coal Conversion, Institute of Coal Chemistry,

Youth Innovation Promotion Association, Chinese Academy of Sciences.

E-mail: qdzhang@sxicc.ac.cn

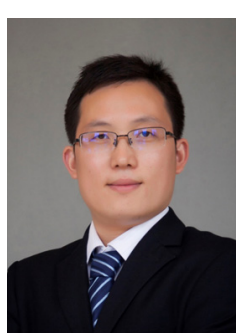

Prof. Hongzhang Zhang (Guest Editor)

Energy Storage Division,

Dalian Institute of Chemical Physics,

Youth Innovation Promotion Association,

Chinese Academy of Sciences.

E-mail: zhanghz@dicp.ac.cn

DOI: 10.1016/S1872-2067(20)63553-7 | http://www.sciencedirect.com/science/journal/18722067 | Chin. J. Catal., Vol. 41, No. 4, April 2020 (C) 2020, Dalian Institute of Chemical Physics, Chinese Academy of Sciences. Published by Elsevier B.V. All rights reserved. 